\section{Enhancing the band gap of an active metamaterial}

\author{
Journal Title \\ $\mathrm{XX}(\mathrm{X}): 2-18$ \\ (C)The Author(s) 2015 \\ Reprints and permission: \\ sagepub.co.uk/journalsPermissions.nav \\ DOI: 10.1177/ToBeAssigned \\ www.sagepub.com/
}

@SAGE

\begin{abstract}
Metamaterials have been the subject of significant interest over the past decade due to their ability to produce novel acoustic behaviour beyond that seen in naturally occurring media. As well as their potential in acoustic cloaks and lenses, of particular interest is the appearance of band gaps which lead to very high levels of attenuation across the material within narrow frequency ranges. Unlike traditional periodic materials which have been employed at high frequencies, the resonant elements within metamaterials allow band gaps to form within the long wavelength limit; at low frequencies where it is most difficult to design satisfactory passive isolation solutions. Hence metamaterials may provide a path to high performance isolation at low frequencies. Passively these band gaps occur over a narrow bandwidth, however the inclusion of active elements provide a method for enhancing this behaviour and producing attenuation over a broad band. A new type of active viscoelastic metamaterial is presented that achieves double negativity and could be employed as a high performance vibration isolator at low frequencies. A mathematical method for manipulating the band gap profile is developed and a prototype is produced. The passive band gap is confirmed in the laboratory, and then by applying active control using optimised feedback filters it is shown that the region at which attenuation occurs around the band gap could be greatly enhanced whilst retaining the peak passive band gap performance. The active metamaterial demonstrates that a unified design philosophy matching the best features of active and passive functionality can achieve high levels of attenuation over wide frequency bands.
\end{abstract}

\title{
Keywords
}

metamaterial, active control, vibration, vibration control 


\section{Introduction}

Acoustic metamaterials have been the subject of significant research activity in recent years due to their ability to produce behaviour outside that of normal media. Locally resonant periodic materials, called metamaterials, were first proposed by Smith and Kroll (2000) in the electromagnetic domain as a way of producing a medium that was 'left handed', exhibiting the behaviour of a material with negative electromagnetic permeability and permittivity, as described by Veselago (1968) many years earlier. More recently, the analogy between acoustic and electromagnetic waves has lead to research in the field of acoustic metamaterials (Fang et al. 2006; Liu et al. 2000), where the analogues of permeability and permittivity are density and bulk modulus respectively. At resonance both of these material parameters can become negative simultaneously. When this happens the material is said to be double negative (DNG), and negative wave refraction occurs due to the refractive index having a negative sign. In this region the group and phase velocity vectors are anti-parallel; the waves are still governed by Snell's law but the refracted wave lies to the opposite side of the incident normal than one would expect in a regular transmission medium. These novel properties have been proposed as a potential solution to achieve acoustic cloaking (Chen and Chan 2010) and subwavelength acoustic lenses (Liu et al. 2000).

In addition to this double negative behaviour, resonance also creates what are known as band gaps in the dispersion characteristics of metamaterials, where high levels of attenuation occur and wave transmission is blocked. Since this attenuation is due to a resonant mechanism, band gaps can be tuned to sub-wavelength frequencies and employed at frequencies much lower than traditional phononic crystals which rely on the Bragg scattering mechanism related to the periodic properties of the material. Since low frequencies are particularly difficult to isolate, the ability to achieve high levels of wave attenuation across the material structure potentially makes metamaterials a powerful new tool in the control of noise and vibration (Cheng et al. 2008).

However, the resonant nature of band gaps mean that attenuation occurs only over a narrow bandwidth and it will therefore be necessary to enhance these properties before metamaterials can be employed in situations where noise sources are broadband or do not remain stationary in frequency. One method used to broaden the bandwidth of the

${ }^{1}$ Institute of Sound and Vibration Research, University of Southampton, UK

\section{Corresponding author:}

Steve Daley, Insititute of Sound and Vibration Research University of Southampton University Road Southampton SO17 1BJ, UK.

Email: s.daley@soton.ac.uk 
band gap using passive techniques is to stagger the natural frequencies of the resonant inclusions such that they each behave at independent, adjacent frequencies (Reynolds et al. 2012; Ding and Zhao 2011). The performance of this technique however is limited by the requirement to have a large number of resonators, and reduces the depth of the band gap (Reynolds et al. 2012). Passive solutions also mean that the frequency range at which attenuation occurs is fixed. Whilst adaptive solutions have been suggested (Lee et al. 2012; Hao et al. 2012) the mechanical nature of these means their reaction speed is relatively slow, and their bandwidth limited.

More recently the concept of active metamaterials has been suggested as a way of controlling and enhancing their behaviour (Baz 2010; Akl and Baz 2010; Pope and Daley 2010; Reynolds et al. 2012; Airoldi and Ruzzene 2011). This has the potential to overcome some of the shortcomings of passive metamaterials. Much of the published work so far has focused on using active elements with passive shunt circuits to achieve effective material parameters impractical using passive methods (Baz 2010; Akl and Baz 2010; Airoldi and Ruzzene 2011). However more recently a metamaterial with a multichannel, fully active architecture that could increase metamaterial performance was suggested by Pope and Daley (2010).

In an earlier paper (Reynolds and Daley 2014) the authors introduced a new type of active viscoelastic metamaterial that was designed to achieve double negative behaviour passively and that possesses a deep resonant band gap. Although an experimental metamaterial was produced by Reynolds and Daley (2014) that demonstrated the potential of the active control architecture, the paper contained only preliminary proof of concept results. Moreover the viscoelastic metamaterial was produced using additive layer manufacturing techniques that limited the passive performance of the isolation device. In this paper a similar device is developed using conventional manufacturing techniques that has a behaviour that is very close to theoretical predictions. Methods for calculating the effective material parameters and to shape the passive band gap were described by Reynolds and Daley (2014) and these are used here to show that by coupling the natural frequency of the resonant elements with the natural frequency of the transmission elements a wide, symmetric band gap can be achieved. This idea is then used to create the prototype active metamaterial, designed to be used as a high performance vibration isolator. Using optimised feedback filters, in both single-channel and multi-channel configurations, it is demonstrated that the bandwidth over which attenuation occurs can be enhanced, greatly improving on the purely passive band gap performance.

Prepared using sagej.cls 


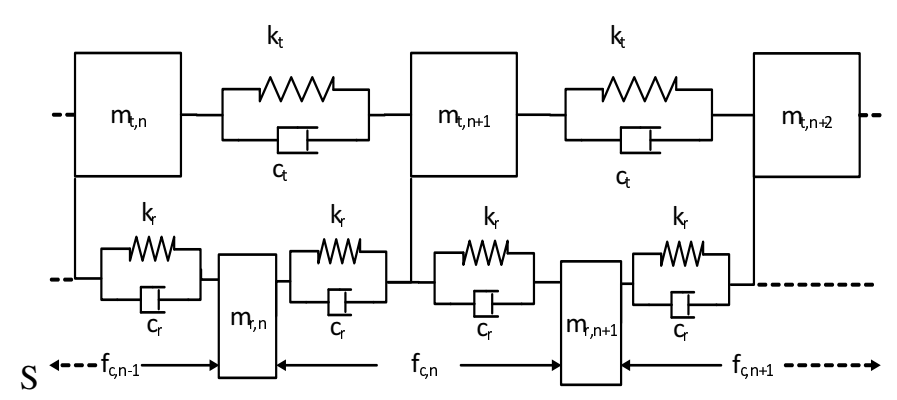

(a)

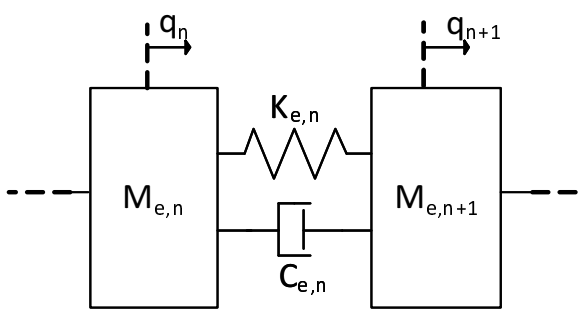

(b)

Figure 1. (a) Lumped parameter model of the active viscoelastic metamaterial. (b) A simple chain of equivalent masses and viscoelastic connections

\section{The active viscoelastic metamaterial}

The active viscoelastic metamaterial model, shown in Fig. 1a, consists of a chain of transmission masses, $m_{t}$, connected by viscoelastic connections represented by stiffness and damping elements, $k_{t}$ and $c_{t}$ respectively. Each transmission mass is then coupled to two resonator masses, $m_{r}$, via additional viscoelastic connections, $k_{r}$ and $c_{r}$. Active forces are applied reactively between adjacent resonator masses, represented by $f_{c}$.

The material can be considered as equivalent to a simple chain of masses connected by viscoelastic connections as shown in Fig. 1b, the equation of motion of which can be written as Eq. 1.

$$
-\omega^{2} M_{e} x_{n}+\left(i \omega C_{e}+K_{e}\right)\left(2 x_{n}-x_{n+1}-x_{n-1}\right)=f_{n}
$$

By formulating and rearranging the equations of motion of the metamaterial model in Fig. 1a into the form of Eq. 1 it can be shown that the effective mass, $M_{e}$ and stiffness 


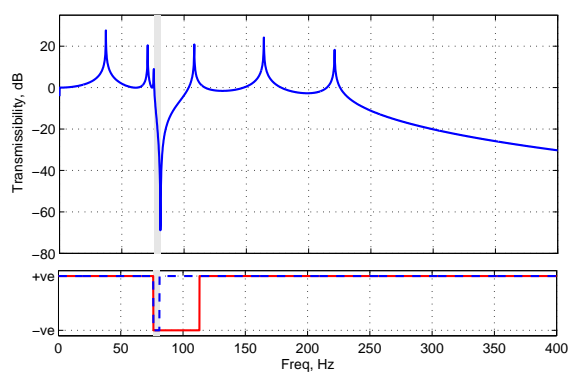

(a)

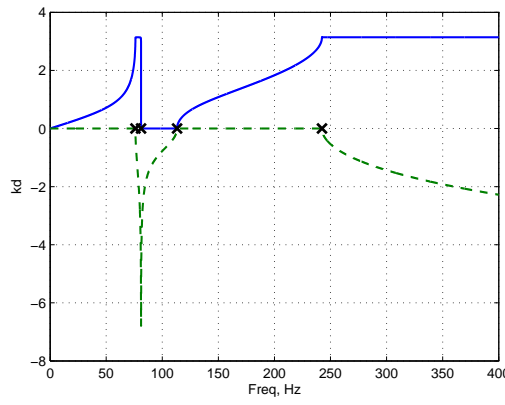

(b)

Figure 2. (a) The transmission response (top) of a typical coupled viscoelastic metamaterial model and (bottom) the sign of the negative effective mass (solid) and stiffness (dashed). The DNG region is shaded grey. (b) The real (solid) and imaginary (dashed) part of the dispersion relationship of the material

and damping $K_{e}+j \omega C_{e}$ of the metamaterial are given by

$$
\begin{gathered}
M_{e}=m_{t}+\frac{2 m_{r}\left(k_{r}+j \omega c_{r}\right)}{-\omega^{2} m_{r}+2\left(k_{r}+j \omega c_{r}\right)} \\
K_{e}+j \omega C_{e}=k_{t}+j \omega c_{t}+\frac{\left(k_{r}+j \omega c_{r}\right)^{2}}{-\omega^{2} m_{r}+2\left(k_{r}+j \omega c_{r}\right)}
\end{gathered}
$$

Unlike other viscoelastic metamaterials from the literature which have negative effective mass (Pope and Daley 2010; Huang and Sun 2009), by coupling the resonator elements to multiple transmission elements the metamaterial presented here can achieve double negativity. These expressions are complex and taking the real part yields the quantities of interest, the effective inertial mass $\operatorname{Re}\left(M_{e}\right)$ and the effective stiffness $K_{e}$. The imaginary parts of these expressions represent the dissipative losses of the system, $\operatorname{Im}\left(M_{e}\right)$ and effective damping $C_{e}$. A typical passive material transmission response (the motion of the last transmission mass related to the first), including the sign of each material parameter is shown in Fig. 2

Previous studies have suggested that the band gap occurs due to the metamaterial being in its single negative region, leading to an imaginary wavenumber, $k$. When the metamaterial is made to be double negative this 'left-handed' material has a negativereal wavenumber and a passband occurs(Lee et al. 2010). However, for the active metamaterial presented here the double negative region coincides with the band gap, as shown in Fig. 2a. As shown in Reynolds and Daley (2014) this can be explained using the Bloch dispersion relationship of a the periodic metamaterial given by Eq. 4, where 


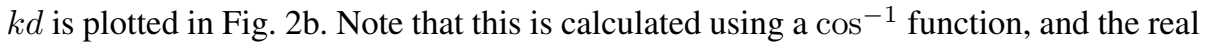
part of the result has a restricted domain of $0 \leq \operatorname{Re}(k d) \leq \pi$. In reality the dispersion relationship can span multiple branches of this function and is not restricted in this way.

$$
\cos k d=1-\frac{\omega^{2} M_{e}}{2\left(K_{e}+j \omega C_{e}\right)}
$$

In the case of the viscoelastic metamaterial the wave number will be complex when $|\cos k d|>1$. Therefore, defining the term $\alpha=\omega^{2} M_{e} /\left(2 K_{e}\right)$, a complex wave number exists where $\alpha>2$ or $\alpha<0$. Using Equations 4, 2 and 3, these two conditions can be used to derive 3 inequalities that define the band gap. Taking the first condition, $\alpha$ will be positive if both the numerator and denominator are of the same sign, and therefore if both of the following inequalities are simultaneously true or false

$$
\begin{aligned}
\omega^{2}<\frac{\left(m_{t}+m_{r}\right) k_{r}}{m_{t} m_{r}} \\
\omega^{2}<\frac{\left(2 k_{t}+k_{r}\right) k_{r}}{k_{t} m_{r}}
\end{aligned}
$$

These expressions represent the numerator and denominator respectively. Taking the second condition where $\alpha>2$, a third inequality can be derived and written as a quadratic expression in $\omega^{2}$

$$
-\omega^{4} m_{t} m_{r}+\omega^{2}\left(2\left(m_{t}+m_{r}\right) k_{r}+4 m_{r} k_{t}\right)-4\left(k_{r}\left(2 k_{t}+k_{r}\right)\right)>0
$$

This has two positive solutions, denoting two key break frequencies

$$
\begin{gathered}
\omega_{0, r}^{2}=\frac{2 k_{r}}{m_{r}} \\
\omega_{U L}^{2}=\frac{2 k_{r}+4 k_{t}}{m_{t}}
\end{gathered}
$$

Now using Eq. 8 with 5 and 6, two more break frequencies can be defined

$$
\begin{aligned}
\omega_{\text {mid }}^{2} & =\omega_{0, r}^{2}\left(1+\frac{k_{r}}{2 k_{t}}\right) \\
\omega_{\text {end }}^{2} & =\omega_{0, r}^{2}\left(1+\frac{m_{r}}{m_{t}}\right)
\end{aligned}
$$

These four frequencies can be used to describe both the location and form of the band gap. The natural frequency of the resonator elements with fixed-fixed boundary conditions is equal to $\omega_{0, r}$, and coincides with the frequency at which Eq. 7 becomes 


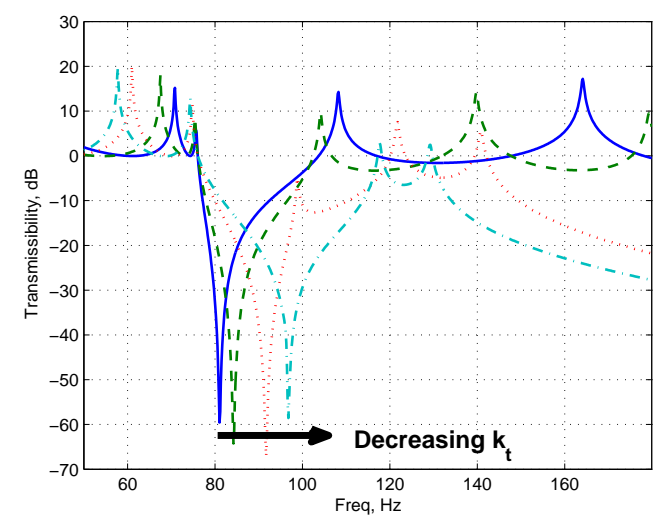

Figure 3. A comparison of the transmissibility 4 metamaterials consisting of 4 layers, with decreasing values of $k_{t}$ causing the band gap centre point to increase in frequency

true. This denotes the lower edge of the band gap. Moving up in frequency causes the left hand side of Eq. 7 to become larger as the denominator described by Eq. 6 becomes smaller, approaching zero at $\omega_{m i d}$; here band gap attenuation peaks as the expression $\omega^{2} M_{e} /\left(2 K_{e}\right)$ resonates. Now as the frequency is increased further $2 K_{e}$ becomes larger and the magnitude of $\alpha$ reduces along with the level of attenuation. The attenuation ceases when Eq. 5 becomes false, defined by the frequency $\omega_{\text {end }}$. The fourth break frequency, $\omega_{U L}$, describes where attenuation begins at high frequencies, when the structure behaves like a low pass mechanical filter. This point is independent of the resonator mass.

This method of wavenumber analysis provides a useful tool in determining the width and form of the resonant band gap, and demonstrates it is possible to adjust the total width of the band gap by adjusting the ratio of the resonator and transmission masses, and the location of the midpoint by manipulating the ratio of the resonator and transmission stiffnesses. The four break frequencies are shown on the dispersion relationship shown in Fig. $2 \mathrm{~b}$ as black crosses. Comparing with Fig. 2a, there is a small discrepancy between the onset of the band gap and high frequency roll off and the calculated values for $\omega_{0, r}$ and $\omega_{U L}$. This is due to the inhomogeneous nature of the finite metamaterial model; the wave number analysis assumes an infinite number of identical layers, and becomes less prominent at higher layer numbers.

In its 'normal' mode of operation, one would expect that the band gap be tuned such that $\omega_{0, r}<\omega_{\text {mid }}<\omega_{\text {end }}<\omega_{U L}$, although this does not need to be the case. However, depending on the location of the band gap in relation to the onset of high frequency rolloff the shape of the band gap changes. Consider the transmissibility of four examples of 
the coupled metamaterial, each where the value for $k_{t}$ is reduced relative to the previous case. The transmissibility of four such materials, each consisting of 4 layers, is shown in Fig. 3. As the figure shows, the onset frequency of the band gap, $\omega_{0, r}$, remains constant, as would be expected from Eq. 8. The mid-point and end-point frequencies however, increase, widening the band gap, and creating a more symmetrical profile. Examining Eq. 11, this increase in band gap end-point appears to contradict the equation for $\omega_{\text {end }}$, however it is important to remember that the calculated break frequencies are for a material of infinite layers and again this effect disappears with higher layer numbers.

A key result is shown by the light blue/dot-dashed response, where the band gap is wide and symmetrical. In this case the metamaterial has been tuned such that $2 k_{r} / m_{r}=2 k_{t} / m_{t}$. This scenario can be considered as being when the natural frequency of a resonant element with fixed-fixed boundary conditions $\left(\omega_{0, r}\right)$ is equal to the natural frequency of a transmission element with fixed-fixed boundary conditions. In a phononic crystal based metamaterial the Bragg scattering based band gap occurs when the wavelength of the incident sound coincides with twice the periodic constant, $\lambda=2 d$. It has been shown by Xiao et al. (2011) among others that when the Bragg gap of a metamaterial is tuned to coincide with the (often narrow and asymmetrical) resonant band gap then the two couple together to create a wide, symmetrical band gap. In the lumped parameter model used for the coupled metamaterial the concept of a periodic constant and wavelength do not apply, since the model has no physical dimensions or sound speed. However, it seems reasonable to equate the natural frequency of a single fixed-fixed transmission cell with the 'natural wavelength' of a single layer of a periodic crystal. Therefore the phenomenon shown here can be considered analogous to the behaviour seen in phononic crystal based metamaterials when the Bragg and resonant band gaps are coupled to create a wide, symmetric 'super' band gap.

\section{Producing the metamaterial}

To test the metamaterial performance in the laboratory a 4 layer prototype was produced from machined steel ${ }^{*}$. The prototype was designed to have the form of a vibration isolator and consists of steel discs separated by rubber springs (fitted post production) acting as the transmission medium, and between each transmission layer is a resonator mass, connected to each transmission layer using leaf springs. The material

* The active metamaterial presented here is covered by a patent (pending) application number PCT/GB2014/050684 


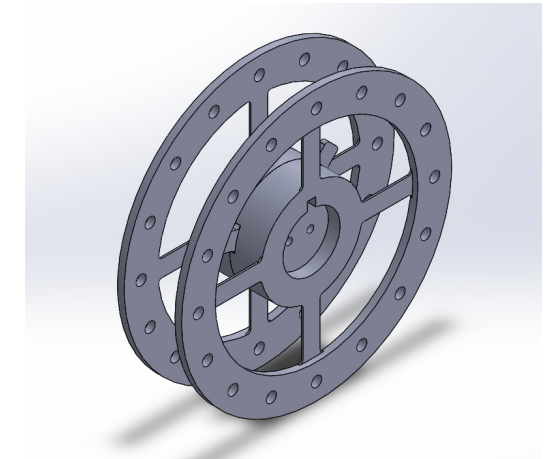

(a)

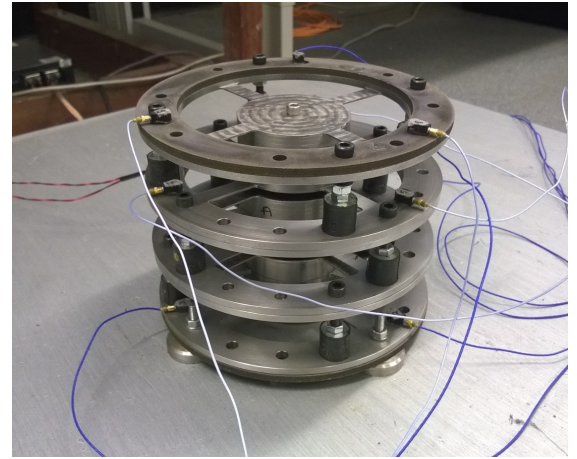

(b)

Figure 4. (a) 3DCAD representation of a cell of the metamaterial design. (b) The assembled metamaterial

was produced as 3 distinct cells that bolt together to produce a continuous periodic 4 layer material. 2 voice coil reactive actuators fitted to the 3 resonator masses to provide an active control force, with the top and bottom resonator accommodating magnet sections and the middle mass having a coil attached top and bottom. A CAD representation of the steel cell design is shown in Fig. 4a, alongside a photo of the assembled material in Fig. 4b.

The metamaterial is fixed to a steel base which is excited using an inertial actuator, the motion of each mass can then be measured using accelerometers and the transmissibility determined. For both the passive and active results presented in this section a dSpace rapid prototyping system is used to provide the excitation signal, record the accelerometer signals and process any control input and output signals.

The open loop transmissibility is determined by calculating the transfer function between the acceleration measured at the first transmission mass to the acceleration at the last transmission mass as a result of a white noise disturbance being applied through the disturbance actuator. The measured transmissibility is shown by the blue line in Fig. 5a. The measured response has a deep resonant band gap situated around $170 \mathrm{~Hz}$, and a high frequency roll-off occurring above $400 \mathrm{~Hz}$ as the material acts as a low pass structural filter. Included in the figure is the predicted transmissibility calculated using the lumped parameter model from Section 2 , given by the dot-dashed line. The modelled and measured transmissibility shows very good agreement with the measured response. The plant response for the two actuators is shown in Fig. 5 b. 


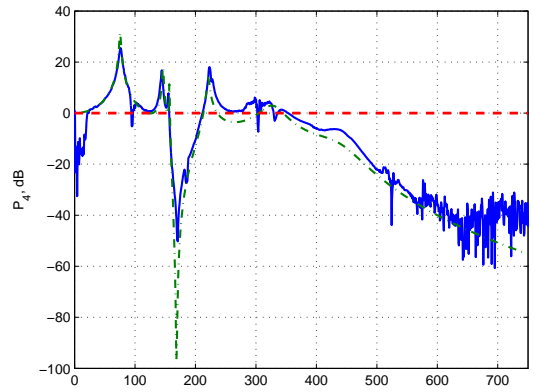

(a)

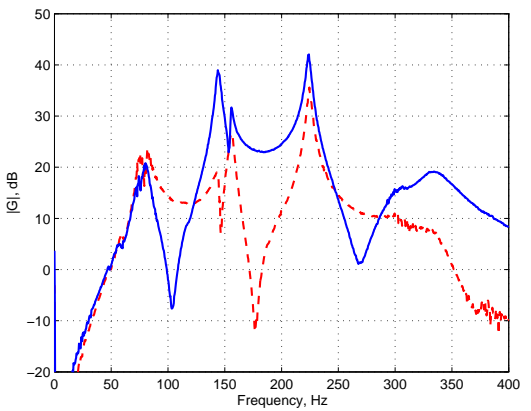

(b)

Figure 5. (a) Measured vertical transmissibility of the metamaterial (solid) compared to the response of the model adapted for best fit (dot-dashed). (b) The measured plant responses $G$ from the inputs of actuator A (solid blue) and actuator B (dotted red) to the final transmission plate.

\section{Enhancing the band gap using active control}

The passive response exhibits a deep resonant band gap achieving over $6 \mathrm{~dB}$ attenuation over a bandwidth of approximately $50 \mathrm{~Hz}$. Next, to increase the bandwidth over which attenuation occurs feedback filters are designed using constrained non-linear optimisation (CNO) performed using the fmincon function that is included as part of the optimisation toolbox for the MATLAB computing environment. This uses an interior point optimisation algorithm based on Coleman and Li (1996); Byrd et al. (1999). Both single-channel (SISO) and multi-channel (MIMO) controllers were investigated, and the controllers were chosen to be FIR filters with the coefficients $\boldsymbol{w}$, thereby ensuring open loop stability. The cost function to be minimised, $\gamma$, is defined as the mean square closed loop transmissibility across a chosen frequency range

$$
\gamma(\boldsymbol{w})=\frac{1}{\omega_{u l}-\omega_{l l}} \sum_{\omega_{l l}}^{\omega_{u l}}|\boldsymbol{P}(j \omega) \boldsymbol{S}(\boldsymbol{w}, j \omega)|^{2}
$$

where the transmissibility is the product of the primary response measured in Fig. 5a, denoted $\boldsymbol{P}(j \omega)$, and the sensitivity function of the control loop $\boldsymbol{S}(j \omega)$. The sensitivity function is defined as $(1+\boldsymbol{G}(j \omega) \boldsymbol{H}(j \omega))^{-1}$ where $\boldsymbol{H}(j \omega)$ and $\boldsymbol{G}(j \omega)$ represent the controller and plant responses respectively. The optimisation was constrained to achieve closed loop stability by ensuring that the generalised Nyquist stability criterion (Maciejowski 1989) was adhered to by ensuring that the following condition was met

$$
\therefore-1<\operatorname{Re}\left(\lambda_{i}(\omega, \boldsymbol{w})\right)
$$


for all $i, w$, where $\lambda_{i}(w)$ represents the $i$ th eigenvalue of $\boldsymbol{G}(j \omega) \boldsymbol{H}(j \omega)$. In addition this stability is made robust to uncertainties in the plant response using the robust stability constraint (Elliott 2000)

$$
\left\|\boldsymbol{T}_{0} B\right\|_{\infty}<1
$$

where $\boldsymbol{T}_{0}$ is the complimentary sensitivity function (Skogestad and Postlethwaite 2005) of the system and $B$ is a multiplicative uncertainty bound. In the sequel for the SISO case, in order to achieve a gain margin of approximately $3.5 \mathrm{~dB}$ and a phase margin of approximately $30^{\circ}$ the multiplicative uncertainty bound was set to $B=0.5$ (Elliott 2000). For the MIMO case the concept of gain and phase margins do not apply, however the multiplicative uncertainty bound was kept at $50 \%$ for consistency.

\section{Applying SISO Controllers}

Initially single channel filters were optimised to use the actuator connected to the final resonator mass, herein referred to as actuator A. One can envisage two use cases for the active isolator, first the suppression of tonal disturbances where the band gap provides the required attenuation of the disturbance tone, but suppression of out of band resonances is desirable either during machine run up or due to out of band noise. Second, to widen the band gap for band-limited disturbances. With this in mind the optimisation routine was applied over four different frequency bands.

- Suppressing the resonance between 50 and $100 \mathrm{~Hz}$

- Extending the band gap over lower frequencies from 70 to $170 \mathrm{~Hz}$

- Extending the band gap over higher frequencies from 200 to $250 \mathrm{~Hz}$

- Simultaneously extending the band gap over both lower and higher frequencies from 100 to $250 \mathrm{~Hz}$

The optimisation routine utilised is computationally expensive. Each iteration requires estimation of the objective function gradient, in this case $\gamma^{\prime}(\boldsymbol{w})$; there are as many gradient estimates as there are filter coefficients. In addition to this the input is in the time domain (i.e. filter coefficients) and the output $\gamma$ is in the frequency domain, requiring a transformation between domains. Also, to ensure the calculated Nyquist stability condition translates to stability in practice at all frequencies the granularity of the frequency bins must remain small, and the length of the FFT must be high. With these factors in mind the filters were designed to have the relatively modest length of 128, using an NFFT of 1024 and a sampling rate of $1.6 \mathrm{kHz}$. The initial coefficients of the filter, $\boldsymbol{w}_{0}$ were set to 0 .

Prepared using sagej.cls 


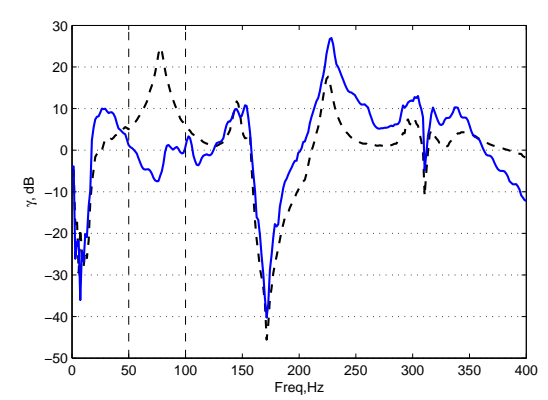

(a)

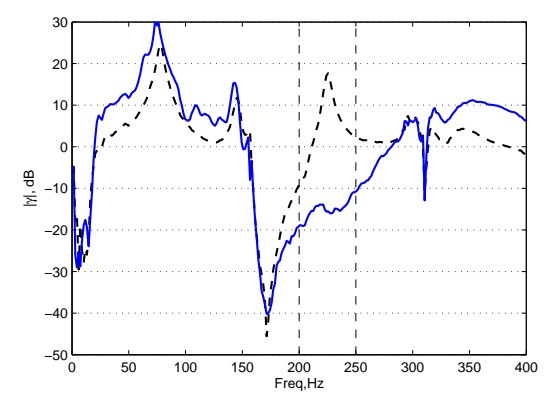

(c)

Figure 6. The measured closed loop transmission coefficient when the filter was optimised over $50-100 \mathrm{~Hz}(\mathrm{a}), 70-170 \mathrm{~Hz}$ (b), $200-250 \mathrm{~Hz}$ (c) and $100-250 \mathrm{~Hz}$ (d), for a 128 coefficient filter (blue solid). The upper and lower frequency bounds of the optimisation are shown by the vertical dotted lines and the open loop transmission coefficient is shown as the black dashed line

The resulting filters were applied to the metamaterial prototype, with the measured closed loop transmission coefficients presented in Fig. 6. The results for the filters optimised over $50-100 \mathrm{~Hz}$ (Fig. 6a) demonstrate that the large resonance in this region can be effectively suppressed, with a peak level of control of over $30 \mathrm{~dB}$. There is enhancement in other areas of the frequency response due to the waterbed effect, including a small narrowing of the passive band gap, however, a large proportion of the passive band gap attenuation remains. Minimising the response in the $70-170 \mathrm{~Hz}$ region (Fig. 6b) also shows that the out of band resonances at $70 \mathrm{~Hz}$ and at $150 \mathrm{~Hz}$ can be effectively suppressed across almost the entire target range. The plant response of actuator A, the solid blue line in Fig. 5b, shows a low degree of coupling into the final transmission plate at frequencies around $100 \mathrm{~Hz}$, meaning a large amount of control effort is required here to influence the motion of the plate. This could potentially limit the performance here in the $70-170 \mathrm{~Hz}$ region, so to achieve effective suppression of 
the out of band resonances is encouraging. The opposite is true in the frequency range above the band gap, where the actuator enjoys a good degree of coupling, meaning that control in the $200-250 \mathrm{~Hz}$ region (Fig. 6c) is very successful, not only suppressing the resonance at $220 \mathrm{~Hz}$ but extending the band gap, doubling the bandwidth over which attenuation of over $10 \mathrm{~dB}$ is achieved from $\sim 50 \mathrm{~Hz}$ to $\sim 100 \mathrm{~Hz}$.

\section{Applying MIMO Controllers}

To design the multichannel controllers an additional error signal was required to ensure the control problem, with its 2 actuators/outputs, remained fully determined (Elliott 2000). Therefore as well as minimising the vibration at the final transmission plate, the optimisation was also set to minimise the vibration at the penultimate transmission plate, using a weighting matrix to prioritise the error signal from the final transmission plate, ensuring the performance of the isolator was not compromised. In addition, to assist convergence a regularisation parameter, $\beta$, was applied to alter the cost function such that what is minimised is not only the magnitude of the error signal but also the weighted mean-square effort term using the control signal $\boldsymbol{u}$ (Elliott 2000). The multichannel cost function was therefore

$$
\gamma(\boldsymbol{w})=\frac{1}{\omega_{u l}-\omega_{l l}} \sum_{\omega_{l l}}^{\omega_{u l}} \boldsymbol{S}(\boldsymbol{w}, \omega)^{H} \boldsymbol{Q S}(\boldsymbol{w}, \omega)+\sum_{\omega_{0}}^{\omega_{N}} \boldsymbol{u}(\boldsymbol{w}, \omega)^{H} \boldsymbol{R} \boldsymbol{u}(\boldsymbol{w}, \omega)
$$

$\boldsymbol{Q}$ and $\boldsymbol{R}$ are weighting matrices, which were heuristically changed with each optimisation attempt to achieve the best results. However a typical set of weighting matrices would consist of a large number corresponding to the error measurement of the final transmission mass and small numbers otherwise, as shown below.

$$
\boldsymbol{Q}=\left[\begin{array}{cc}
10^{-3} & 0 \\
0 & 10^{3}
\end{array}\right] \quad \boldsymbol{R}=\beta\left[\begin{array}{ll}
1 & 0 \\
0 & 1
\end{array}\right]=\left[\begin{array}{cc}
10^{-6} & 0 \\
0 & 10^{-6}
\end{array}\right]
$$

The multichannel controllers were designed to address the wideband scenario tested in the single channel case, targeting the transmissibility between $100-250 \mathrm{~Hz}$. Three different configurations were tested - a fully populated 2-in 2-out controller consisting of 4 FIR filters, referred to as 'centralised'; a second controller with a diagonal structure consisting of 2 FIR filters, with zeros at the off-diagonal elements, referred to as 'decentralised'; and a final configuration which the same structure as the decentralised example, however the controller consists of 2 identical FIR filters, 
referred to as the 'periodic' controller. The three structures are shown in Table 1, where the subscript $a / b$ and 3/4 represent actuator ' $a$ ' or ' $b$ ' and the 3rd and 4th (final) transmission mass respectively. The three structures were chosen to represent a range of different design approaches. The centralised controller offers the greatest flexibility however as the metamaterial is extended to greater layers may become impractical. The decentralised control scheme could address this, with each layer of the metamaterial having its own discrete control loop. Finally, staying true to the periodic definition of a metamaterial, the periodic controller means each section is both physically and electronically identical to the others, perhaps in the future a modular design could lead to identical cells mass produced on a small scale and used as a building block of an isolation or wave guide scheme.

\begin{tabular}{|l|l|l|}
\hline Centralised & Decentralised & Periodic \\
\hline $\boldsymbol{H}=\left[\begin{array}{ll}H_{b, 3} & H_{b, 4} \\
H_{a, 3} & H_{a, 4}\end{array}\right]$ & $\boldsymbol{H}=\left[\begin{array}{cc}H_{b, 3} & 0 \\
0 & H_{a, 4}\end{array}\right]$ & $\boldsymbol{H}=\left[\begin{array}{cc}H & 0 \\
0 & H\end{array}\right]$ \\
\hline
\end{tabular}

Table 1. The three MIMO controller structures to be designed

Figure 7 presents the measured results for the three controller structures. The periodic filter structure, Fig. 7c, successfully suppresses the resonances either side of the band gap, and achieves attenuation in the higher frequencies of the target region of approximately $5-7 \mathrm{~dB}$. Overall in the lower frequencies the attenuation is lower, at $1-3 \mathrm{~dB}$. The centre of the band gap is preserved, however the overall passive band gap profile is narrowed somewhat. This performance is comparable to, if slightly outperformed by, the single channel case. This is perhaps to be expected; having to apply two identical feedback filters feeding two actuators with different plant responses could be considered a more onerous constraint than designing a single filter and making the second feedback signal zero. Removing the periodic constraint leads to a large improvement in the performance of the metamaterial, as shown in the results for the decentralised controller, Fig. 7b. Here again the centre of the band gap is conserved, and the attenuation across the vast majority of the rest of the targeted frequency range is $7-10 \mathrm{~dB}$. The small exception is the region at approximately $145 \mathrm{~Hz}$, corresponding to the resonant peak at the lower edge of the band gap, where attenuation drops to approximately $2 \mathrm{~dB}$. This control structure shows great potential if employed in an isolation scenario.

Prepared using sagej.cls 


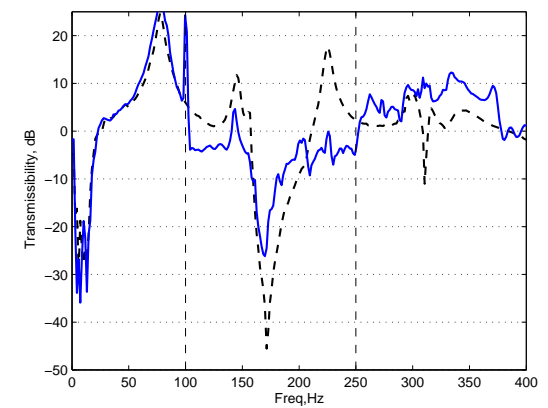

(a)

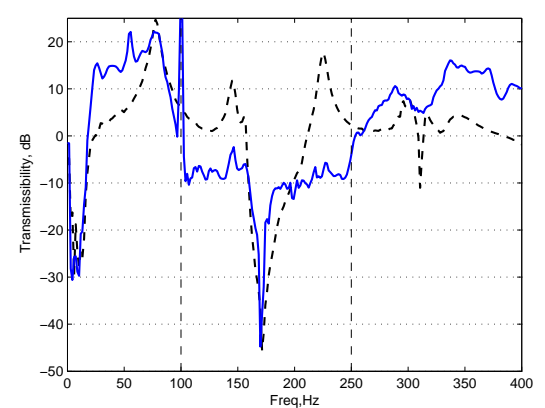

(b)

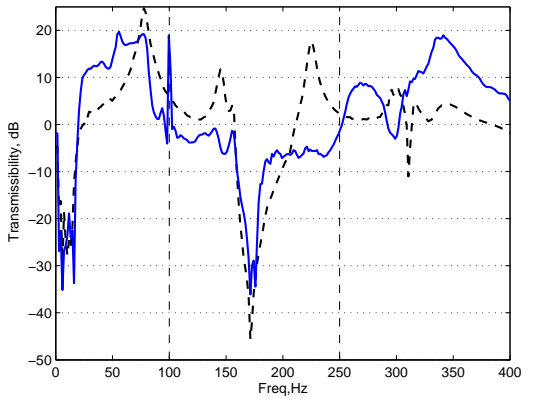

(c)

Figure 7. The passive (blue solid) and active (black dashed) measured transmissibility of the viscoelastic metamaterial when 128 coefficient filters were optimised over $100-250 \mathrm{~Hz}$, and the MIMO feedback controller has a (a) centralised, (b) decentralised and (c) periodic structure

The results for the centralised controller fails to achieve the performance of the previous two examples. It would be expected that since this configuration has the most flexibility it would produce the best results; at the very least the optimisation should be able to produce results equal to the decentralised case by converging to the same solution, with the off diagonal elements remaining at 0 . When applying the optimisation routine to this scenario the fmincon function often would not converge. To assist convergence the initial filter coefficients were then set to the final decentralised solution; a subset of the possible solutions to the centralised problem. This is therefore the minimum one would expect the centralised scenario to achieve; however even then the algorithm would diverge from this solution and fail. By adjusting the weighting matrices of the cost function heuristically a solution was eventually achieved. The metamaterial is a resonant structure and it is thought that 
poor conditioning may be the reason for the failure of the function to converge to a satisfactory solution.

\section{Conclusion}

This paper presents a new type of active viscoelastic metamaterial that is designed to be double negative and have a deep resonant band gap. A method has been developed for manipulating the band gap profile based on four key frequencies and it is shown that by coupling the natural frequency of the resonator and transmission units a wide symmetric 'super' band gap can be achieved. A prototype of the active metamaterial was then produced and its transmissibility was shown to match that of the theoretical model. Next it was shown that by using optimised feedback filters through the inbuilt actuators that out of band resonances could be eliminated and the region at which attenuation occurs around the band gap could be greatly enhanced whilst retaining the peak passive band gap performance. The results here demonstrate that a unified design process that matches the best features of both active and passive functionality can provide large levels of attenuation over wide bandwidths, and show that active metamaterials offer great potential in isolation applications.

\section{Acknowledgements}

This work was funded by the Engineering and Physical Science Research Council (EPSRC) and BAE Systems under a Collaborative Award in Science and Engineering (CASE) award, this support is gratefully acknowledged.

\section{References}

Airoldi L and Ruzzene M (2011) Design of tunable acoustic metamaterials through periodic arrays of resonant shunted piezos. New Journal of Physics 13.

Akl W and Baz A (2010) Configurations of active acoustic metamaterial with programmable bulk modulus. Active and Passive Smart Structures and Integrated Systems 2010, Pts 1 and 27643.

Baz AM (2010) An active acoustic metamaterial with tunable effective density. Journal of Vibration and Acoustics-Transactions of the Asme 132(4).

Byrd RH, Hribar ME and Nocedal J (1999) An interior point algorithm for large-scale nonlinear programming. Siam Journal on Optimization 9(4): 877-900.

Prepared using sagej.cls 
Chen HY and Chan CT (2010) Acoustic cloaking and transformation acoustics. Journal of Physics D-Applied Physics 43(11).

Cheng Y, Xu JY and Liu XJ (2008) Broad forbidden bands in parallel-coupled locally resonant ultrasonic metamaterials. Applied Physics Letters 92(5).

Coleman TF and Li YY (1996) An interior trust region approach for nonlinear minimization subject to bounds. Siam Journal on Optimization 6(2): 418-445.

Ding CL and Zhao XP (2011) Multi-band and broadband acoustic metamaterial with resonant structures. Journal of Physics D-Applied Physics 44(21).

Elliott S (2000) Signal Processing for Active Control. Elsevier Science.

Fang N, Xi DJ, Xu JY, Ambati M, Srituravanich W, Sun C and Zhang X (2006) Ultrasonic metamaterials with negative modulus. Nature Materials 5(6): 452-456.

Hao LM, Ding CL and Zhao XP (2012) Tunable acoustic metamaterial with negative modulus. Applied Physics a-Materials Science \& Processing 106(4): 807-811.

Huang HH and Sun CT (2009) Wave attenuation mechanism in an acoustic metamaterial with negative effective mass density. New Journal of Physics 11.

Lee KJB, Jung MK and Lee SH (2012) Highly tunable acoustic metamaterials based on a resonant tubular array. Physical Review B 86(18).

Lee SH, Park CM, Seo YM, Wang ZG and Kim CK (2010) Composite acoustic medium with simultaneously negative density and modulus. Physical Review Letters 104(5).

Liu ZY, Zhang XX, Mao YW, Zhu YY, Yang ZY, Chan CT and Sheng P (2000) Locally resonant sonic materials. Science 289(5485): 1734-1736.

Maciejowski J (1989) Multivariable feedback design. Electronic systems engineering series. Addison-Wesley. ISBN 9780201182439.

Pope SA and Daley S (2010) Viscoelastic locally resonant double negative metamaterials with controllable effective density and elasticity. Physics Letters A 374(41): 4250-4255.

Reynolds M and Daley S (2014) An active viscoelastic metamaterial for isolation applications. Smart Materials and Structures 23(4): 045030. URL http://stacks.iop.org/ $0964-1726 / 23 / i=4 / a=045030$.

Reynolds M, Daley S, Gao Y, Humphrey V and Pope SA (2012) Controller architectures for optimum performance in practical active acoustic metamaterials. Proceedings of Acoustics 2012: 11th Congres Francais d'Acoustique and 2012 IOA annual meeting. Nantes, France.

Skogestad S and Postlethwaite I (2005) Multivariable feedback control: analysis and design. John Wiley.

Smith DR and Kroll N (2000) Negative refractive index in left-handed materials. Physical Review Letters 85(14): 2933-2936. 
Veselago V (1968) The electrodynamics of substances with simultaneously negative values of permittivity and permeability. Soviet Physics Uspekhi-Ussr 10(4): 509-514.

Xiao Y, Mace BR, Wen JH and Wen XS (2011) Formation and coupling of band gaps in a locally resonant elastic system comprising a string with attached resonators. Physics Letters A 375(12): 1485-1491. 